(z. B. «Medienkritik»)? Was sind ursächliche Bedingungen für das Phänomen (z. B. «Unzufriedenheit mit Mainstreammedien»)? Welche intervenierenden Bedingungen gibt es (z. B. «Wandel des Mediensystem»)? Was ist der Kontext (z. B. «Journalismusverständnis»)? Welche Strategien gibt es (z. B. «Oppositionelle Rhetorik»)? Und schliesslich: Was sind die Konsequenzen (z. B. «Aufruf zum Protest») (Strauss \& Corbin, 1990, S. 96-115). Der dritte Analyseschritt des selektiven Kodierens (3) beschäftigt sich nun abschliessend mit den im vorangehenden Schritt identifizierten Schlüsselkategorien, anhand derer die Theorie herausgearbeitet werden kann und in die alle weiteren Kategorien integriert werden können. Dabei werden nur mehr die für die Theorie relevanten Kategorien betrachtet und miteinander in Bezug gesetzt (Strauss \& Corbin, 1990, S. 116-134).

Der Ansatz der Grounded Theory eignet sich für die Bearbeitung respektive Beantwortung der zweiten Forschungsfrage deswegen besonders gut, um das Phänomen «alternative Nachrichtenmedien» explorativ zu erforschen. Der phänomenologische Zugang ermöglicht es, das Selbstverständnis der Untersuchungsobjekte aus deren Sichtweise zu identifizieren. Von einer normativen Betrachtung wird bewusst Abstand genommen, um erstens eine intersubjektive Nachvollziehbarkeit zu gewährleisten. Zweitens könnte man Gefahr laufen, den Untersuchungsgegenstand in «gute» versus «schlechte» Nachrichtenmedien zu unterscheiden bzw. diese zu bewerten. Dies soll nicht Zweck dieser Analyse sein. Weiter zielt die Grounded Theory auf die Theorieentwicklung ab. Auch hinsichtlich dieses Punktes überzeugt der methodologische Zugang zur Beantwortung der Forschungsfrage. Schliesslich soll einerseits der Begriff «alternative Nachrichtenmedien» eindeutig definiert und weiter das Phänomen differenzierter betrachtet werden, indem unterschiedliche Typen von Alternativmedien herausgearbeitet werden. In diesem Sinne kann von einer theoretischen Weiterentwicklung gesprochen werden.

Für die qualitativen Auswertungen wurde unterstützend das Tool MAXQDA herangezogen. Über das «Web Collector» Add-on des Browsers Google Chrome konnten die zu analysierenden Websites statisch abgespeichert und direkt in die Analysesoftware importiert werden. Somit konnte der Kodierprozess übersichtlicher gestaltet und vereinfacht werden.

\title{
VI.3 Datenerhebung auf Twitter
}

Eines der wesentlichen Ziele dieser Forschung besteht darin, die Netzwerke alternativer Nachrichtenmedien zu untersuchen. Um einen strukturellen Blick auf die Relationen von und gegenüber alternativen Nachrichtenmedien zu erhalten, können (quantitative) Netzwerkanalysen durchgeführt werden. Der quantitative Ansatz hilft dabei, einen ersten, deskriptiven Überblick über die Netzwerk- 
strukturen zu gewinnen. Unter der Vorannahme, dass sich im Zuge des digitalen Strukturwandels Gegenöffentlichkeiten wie alternative Nachrichtenmedien und andere Laienkommunikator_innen ihre Netzwerke vor allem über soziale Medien (bzw. soziale Netzwerke) aufbauen, eignen sich Analysen auf Social-Media-Plattformen zur Beantwortung der Forschungsfrage. Auch die technisch-methodischen Möglichkeiten, derartige Analysen durchzuführen, gehen Hand in Hand mit der Etablierung diverser Plattformen. In der aktuellen Forschung wird die Plattform Twitter bevorzugt genutzt, da die Datenerhebung via API-Schnittstelle relativ problemlos möglich ist - z. B. im Vergleich zu Facebook. Doch nicht nur aus diesem technisch-pragmatischen Grund eignet sich Twitter besonders gut für die Analyse von Netzwerkstrukturen auf Social Media. Wie auch in der Literatur belegt, kann Twitter als Netzwerk für Eliten aus Politik und Journalismus betrachtet werden (Rauchfleisch \& Metag, 2016). Demzufolge wird das Netzwerk in hohem Masse zur Verbreitung bzw. Kommentierung von Nachrichten genutzt - sowohl von professionellen Akteur_innen als auch von Lai_innen. Twitter wird entsprechend als idealer empirischer Wirklichkeitsausschnitt für dieses Projekt betrachtet.

Da die Forschungsfragen $\mathrm{FF}_{3}$ und $\mathrm{FF}_{4}$ auf die Beziehungsnetzwerke alternativer Nachrichtenmedien untereinander, aber auch auf Relationen zu professionellen Nachrichtenmedien und weiteren Akteur_innen des öffentlichen Lebens fokussieren, musste zunächst festgelegt werden, mit welchen Suchbegriffen Daten aus der Twittersphäre erhoben werden. Dahingehend waren zwei Dinge zu berücksichtigen: erstens die technischen Möglichkeiten, um Twitterdaten zu sammeln, und zweitens die Funktionsweisen der Plattform, um deren Logiken entsprechend die idealen Suchbegriffe zu definieren. Twitter erlaubt mittels seiner API-Schnittstelle unterschiedliche Wege, Daten zu erheben. Um überhaupt auf Twitterdaten über diese Schnittstelle zugreifen zu können, muss der/die Forscher_in einen üblichen Twitter-Account besitzen und zusätzlich als sogenannter «Twitter Developer» ${ }^{3}$ registriert sein. Prinzipiell ist es allen Twitter-Nutzer_innen möglich, Developer zu werden, sofern der Zweck und die Ziele hinter der geplanten Datenanalyse offengelegt werden. Nach einer kurzen Prüfung durch Twitter ist es dem/der Nutzer_in möglich, Applikationen zu erstellen, über die Zugangscodes (sogenannte «Tokens») kreiert werden, die zur Verbindung mit der API-Schnittstelle benötigt werden. Für dieses Projekt wurde die Programmiersprache $\mathrm{R}^{4}$ unter Verwendung der Benutzeroberfläche RStudio genutzt, um die Verknüpfung mit Twitter herzustellen und schliesslich Daten zu sammeln. Nach diesem ersten technischen Schritt stellt sich die Frage, mit welcher Funktion und welchen Suchbegriffen operiert wird. Grundsätzlich bestehen mehrere

3 https://developer.twitter.com

4 https://www.r-project.org 
Möglichkeiten, Daten über Twitter zu beziehen. Für diese Studie war es relevant, eine grosse Zahl an Daten in Echtzeit herunterzuladen, weswegen auf die «Stream»-Funktion zurückgegriffen wurde, die unter Verwendung des Packages «streamR» über R durchgeführt werden kann. Mit dieser Funktion können bis zu 400 Suchbegriffe in der Twittersphäre gesucht werden. Tweets, in denen diese Begriffe vorkommen, werden automatisch mit den jeweiligen Zusatzinformationen (wie Screenname, Datum, Anzahl an Retweets etc.) abgespeichert. Bei der Überlegung, welche Suchbegriffe verwendet werden sollen, um ein möglichst vollumfängliches Ergebnis zu erzielen, müssen die Funktionsweisen des Social Networks reflektiert werden. Typisch für Twitter ist die Bezugnahme auf Themen in Form von sogenannten Hashtags (z. B. \#Lügenpresse). Ebenso können weitere Twitter-User über das @-Zeichen verlinkt (z. B. @EpochTimes) oder Beitrage von anderen Twitter-Nutzern retweetet werden. Bei der Verwendung der beiden Symbole \# und @ inklusive Suchbegriffen würden jedoch im Zuge des Trackings viele Posts ausgeschlossen, in denen zwar (alternative) Nachrichtenmedien thematisiert werden, die aber nicht mit Hashtag oder Personenverlinkungen versehen sind. Aus diesem Grund wurde eine Suche der jeweiligen URLs alternativer und professioneller Nachrichtenmedien gewählt, da (insbesondere, wenn es um die Verbreitung von Nachrichten geht) davon auszugehen ist, dass ein grosser Teil der für diese Studie relevanten Tweets mit URL versehen sind, z. B. um auf einen Artikel aus den Medien hinzuweisen. Auch Retweets werden durch diese Samplingmethode miterhoben. Zusätzlich wurden die Twitter-Screennames (sofern vorhanden) der einzelnen Alternativmedien, die auf Twitter vertreten sind, als Suchbegriffe ergänzt ${ }^{5}$.

Insgesamt wurden vier Twitter-Streams aufgesetzt: Für alle im ersten empirischen Schritt bereits gesammelten alternativen Nachrichtenmedien im DACHRaum sowie für jedes der drei Länder ein eigener Stream, um Tweets mit den URLs der professionellen Nachrichtenmedien («Mainstreammedien») zu erfassen ${ }^{6}$. Die Streams liefen über separate, externe Server und die erfassten Tweets wurden jeweils zweistündlich zwischengespeichert, um mögliche Datenausfälle (z. B. aufgrund eines Serverzusammenbruchs) zu minimieren. Insgesamt wurden die URLs von 178 alternativen Nachrichtenmedien aus dem deutschsprachigen Raum gesucht sowie zusätzlich 120 Screennames; 302 URLs deutschsprachiger profes-

5 Die Suche der Screennames musste im Rahmen der Suche der professionellen Nachrichtenmedien ausgeschlossen werden, da ansonsten die Limitierung von 400 Suchbegriffen (keywords) überschritten worden wäre. Da die Suche über URLs ein umfangreicheres Tracking ermöglicht als nur über die Screennames, wurde dieses Verfahren gewählt.

6 In weiterer Folge wird der Begriff «professionelle Nachrichtenmedien» zur Beschreibung von «Mainstreammedien» aus dem DACH-Raum genutzt. Dies bedeutet nicht, dass nicht auch alternative Nachrichtenmedien professionell auftreten und agieren können. 
sioneller Nachrichtenmedien; 94 URLs österreichischer professioneller Nachrichtenmedien; sowie 253 URLs professioneller Nachrichtenmedien aus der deutschsprachigen Schweiz. Die Listen der «Mainstreammedien» aus den drei Ländern wurden induktiv erstellt, mit einem Fokus auf Leitmedien aus dem Bereich Presse (regionale, überregionale, Tages-, Wochenzeitungen, Nachrichten-Magazine) wie auch Online-Nachrichtenmedien und TV-Angebote, die online Nachrichten aufbereiten. Dabei erfolgte keine Einschränkung auf politische Berichterstattung; vielmehr sollte ein vollumfängliches Bild der Nachrichtenberichterstattung im deutschsprachigen Raum geschaffen werden, sei es aus dem Bereich Politik, Wirtschaft oder z. B. auch Sport, IT etc. Ausgeschlossen wurden Magazine mit einem bestimmten Themenfokus, in denen keine aktuellen Nachrichten verbreitet werden. Dabei konnte einerseits auf eine bereits bestehende Liste mit Schweizer Nachrichtenmedien aus Studien des Forschungszentrums Öffentlichkeit und Gesellschaft (fög) der Universität Zürich zurückgegriffen werden. Diese wurde zusätzlich über weitere Quellen induktiv ergänzt (Linker.ch, 2019; Statista, 2019b; WEMF, 2019; Wikipedia, 2019d). Ebenso über Schneeballverfahren wurden die Listen für Deutschland (Netzbeitrag, 2019; Statista, 2019a; Wikipedia, 2019a, 2019b) und Österreich (ARGE Media-Analysen, 2018; Österreichische Auflagenkontrolle, 2018; Österreichische Webanalyse, 2018; Statista, 2019c; Wikipedia, 2019c) gänzlich neu erstellt (vgl. Anhang 1b).

Zur Datenerhebung wurde der Zeitraum zwischen dem 15. September 2019 bis einschliesslich 27. Oktober 2019 gewählt. Diese sechs Wochen eignen sich vor allem deshalb als empirischer Ausschnitt zur Beantwortung der Forschungsfrage, da in ebendiesem Zeitraum bedeutsame politische Wahlen in allen drei Untersuchungsländern stattfanden: Die Nationalratswahl in Österreich am 29. September 2019, die Schweizer Parlamentswahl am 20. Oktober 2019 sowie die Landtagswahl in Thüringen in Deutschland am 27. Oktober 2019. Dadurch, dass die Datenerhebung bereits am 15. September begann, wurde auch der österreichische Wahlkampf - zwei Wochen vor der Nationalratswahl - abgedeckt. Der Erhebungszeitraum rund um politische Wahlen eignet sich deshalb für dieses Projekt, da davon auszugehen ist, dass sich alternative Medien vor allem über die politische Berichterstattung definieren, zumal sie sich (u. a.) als Opposition zur politischen Elite betrachten.

Insgesamt wurden Daten über 43 Tage (1'032 Stunden pro Twitter-Stream) hinweg gesammelt. Ausnahmsweise wurden die Streams - vermutlich aufgrund von Datenüberlastung - automatisch unterbrochen, weshalb vereinzelte Zeiträume nicht in den Datensätzen enthalten sind. Hierbei handelte es sich allerdings jeweils nur um wenige Stunden, die gemessen am gesamten Erhebungszeitrum 
unter 2,7\% liegen ${ }^{7}$. Die erhobenen Datensätze enthielten neben den Tweets als Textdaten zudem weitere Spalten mit zusätzlichen Informationen, in denen beispielsweise der Zeitpunkt der Erstellung oder der/die Autor_in des Tweets sowie die in den Tweets eingebetteten URLs aufgenommen wurden - die schliesslich Grundlage für die Datenerhebung waren. Zusätzlich mussten jene Spalten ergänzt werden, die beispielsweise die URLs von Retweets beinhalten. Im Zuge der Datenbereinigung war es demnach notwendig, einzelne, gekürzte URLs zu unshorten (z. B. «bit.ly»), sodass die URLs in ihrer vollständigen Form analysiert werden konnten. Weiter wurden die Daten unter Verwendung von R mit Regex-Patterns automatisch bereinigt, um potenzielle «false positives» $\mathrm{zu}$ entfernen, also jene Tweets, die irrtümlicherweise aufgrund von Übereinstimmung der Suchterme, aber ohne inhaltliche Übereinstimmung mit den URLs miterhoben wurden ${ }^{8}$. Weitergearbeitet wurde mit zwei Datensätzen (einem Datensatz basierend auf dem Stream alternativer Nachrichtenmedien $\left(n=388^{\prime} 237\right)$ sowie einem kompletten Datensatz inklusive der Mainstreammedien aus Deutschland, Österreich und der deutschsprachigen Schweiz $\left[n=1^{\prime} 424^{\prime} 928^{\circ}\right]$ ), um die geplanten Netzwerkanalysen durchführen zu können. Der Datensatz der alternativen Nachrichtenmedien beinhaltet Tweets, in denen die URLs der gelisteten Newssites sowie Tweets implementiert wurden, in denen die Screennames alternativer Nachrichtenseiten erwähnt wurden. Der Gesamtdatensatz enthält Tweets, in denen URLs entweder von alternativen Nachrichtenseiten oder von «Mainstream»-Nachrichtenseiten eingefügt wurden. ${ }^{10}$ Das geplante Retweet-Netzwerk kann mithilfe des Datensatzes berechnet werden. Für das Follower-Netzwerk müssen weitere Daten erhoben werden. So müssen zuerst alle User_innen, die im Zeitraum getwittert haben, als Unique Users aus dem Datensatz extrahiert werden, um in einem weiteren Schritt deren Follower_innen (also Personen, die diese User_innen via Twitter abonniert haben) herunterzuladen. Auch hierfür wird eine Verbindung zur Twitter-API-Schnittstelle benötigt. Die Erhebung von Followerbeziehungen der Twitter-Accounts ist an gewisse Bedingungen geknüpft: Zum einen können sich nur Personen mit ihrem eigenen Twitter-Login Zugang zur API-Schnittstelle

7 Der für die vorliegende Arbeit bedeutsamste Datensatz mit einem Fokus auf alternative Nachrichtenmedien ist nahezu komplett, mit einem Datenausfall von etwa 0,17\%.

8 So wurden beispielsweise im Stream österreichischer Nachrichtenseiten viele false positives identifiziert, die über das Such-Keyword «tt.com» (Tiroler Tageszeitung) miterhoben wurden.

9 Deutschland ( $\left.n=717^{\prime} 668\right)$, Österreich $\left(n=118^{\prime} 491\right)$, Schweiz $(n=370 ' 502)$, alternative Nachrichtenmedien (basierend auf den URLs: $n=218^{\prime} 267$ ).

10 Im Gesamtdatensatz wurden keine zusätzlichen Tweets herangezogen, in denen die Screennames der Medien erwähnt wurden. Dies deshalb, da im Stream der deutschen Medien eine Suche der Screennames nicht möglich war, da ansonsten das Limit an Keywords erreicht worden wäre. Durch die Bereinigung der Daten ausschliesslich basierend auf den URL-Keywords ist eine bessere Vergleichbarkeit der Daten garantiert. 
verschaffen. Weiter wird ein Twitter-Developer-Account (https://developer.twitter.com) benötigt, der mit dem persönlichen Account verknüpft wird. Durch die verpflichtende Angabe und Bestätigung an Twitter, wofür die Daten verwendet werden (z. B. wissenschaftliche Forschung), soll eine missbräuchliche Verwendung des Zugangs verhindert werden. Sobald der Account freigeschaltet wird, kann eine «App» erstellt werden, mit deren Hilfe ein Zugang (via «API key» und «access token») zur Twitter-Datensphäre ermöglicht wird. Auch die Datenerhebung selbst ist an Limitierungen gebunden. Pro Stunde können maximal 60 User-Informationen heruntergeladen werden. Die Aufbereitung der Daten erfolgte unter Verwendung von R (Package: igraph), für die Community-Detection (um unterschiedliche Cluster innerhalb der Netzwerke zu identifizieren) und für die Visualisierung wurde das Tool Gephi unter Rückgriff auf den Louvain-Algorithmus (Blondel, Guillaume, Lambiotte \& Lefebvre, 2008) genutzt. Der Louvain-Algorithmus ist ein hierarchischer Clustering-Algorithmus und eignet sich als effiziente Methode, da er stabile Ergebnisse respektive Modularitätsscores erzielt (Lancchinetti \& Fortunato, 2009).

\section{VI.4 Relationale Analyse der Digitalplattform Twitter}

Die im vorherigen Schritt erstellten Netzwerke stellen die Basis für die letzte empirische Untersuchung dar. In Anlehnung an den Ansatz der relationalen Soziologie (vgl. Kapitel IV.1) wird davon ausgegangen, dass Netzwerke nicht aus starren Strukturen bestehen, sondern vor allem auf Narrationen, also dem Dazwischenliegenden im Sinne von sozialen Mustern, basieren (Emirbayer, 1997; Häußling, 2010b). Dieser Logik folgend wird im Rahmen dieser Studie versucht, quantitative und qualitative Methoden bestmöglich zu kombinieren, um die jeweiligen Stärken auszuschöpfen bzw. etwaige methodische Schwächen zu minimieren. Es wird angenommen, dass der letzte empirische Schritt dieser Studie die vorangehenden nutzen kann, um ein abschliessendes Bild über das zu untersuchende Phänomen alternativer Nachrichtenmedien als Gegenöffentlichkeiten gewährleisten zu können. So kann insbesondere die bereits erstellte typologische Darstellung alternativer Nachrichtenmedien für die Interpretation einzelner im Zuge der Netzwerkanalyse identifizierter Communities genutzt werden. Weiter sollen die visualisierten Netzwerke und vor allem Beziehungen zwischen Akteur_innen detaillierter beschrieben und interpretiert werden. Eine quantitative Netzwerkanalyse stösst hier an ihre Grenzen. Qualitative Auswertungsmethoden können an diesem Schritt ansetzen, um grosse Datenmengen inhaltlich zu interpretieren. Dies erfolgt zum Teil schon bei der Interpretation der Communities, für die einzelne Knotenpunkte inhaltlich eingeordnet werden müssen, z. B. durch einen Blick auf den jeweiligen Twitter-Account. Der letzte empirische Schritt möchte aber auch 